\title{
A Phase Space Approach to Gravitational Entropy
}

\author{
Tony Rothman \\ Dept. of Physics, Illinois Wesleyan University
}

July 15, 2021

\begin{abstract}
We examine the definition $S=\ln \Omega$ as a candidate function for "gravitational entropy." We calculate its behavior for gravitational and density perturbations in closed, open and flat cosmological models and find that in all cases it increases monotonically. We are also able to calculate the entropy density of gravitational radiation produced by inflation. We compare the results with the behavior of the Weyl-tensor squared. Applying the formalism to black holes has proved more problematical.
\end{abstract}

It is appropriate that I speak today on the subject of gravitational entropy because it was George Ellis, on my last visit to Cape Town, who instigated my investigations into this areathough he shouldn't be held responsible for any of the results.

The problem is well-known. Ordinary thermodynamic systems, a box of gas for example, tend to grow more homogeneous with 
time, whereas gravitating systems tend to become more inhomogeneous with time. In this sense gravitating systems are "antithermodynamic." I'm sure you've all seen the picture in Roger Penrose's book that shows this behavior. Indeed, the tendency toward inhomogeneity of a gravitating system can be viewed as a manifestation of the long-range nature of the gravitational force, which tends to cause the components of the system to clump.

Now, ordinarily, for thermodynamic system, we associate the increase of homogeneity with an increase in thermodynamic entropy. This is a sign convention. One could, after all, choose Boltzmann's original $\mathrm{H}$ as the entropy function, and it would decrease with homogeneity. Whichever sign one chooses, though, "gravitational entropy" or the "gravitational arrow of time" points in the opposite direction to the thermodynamic arrow.

The question then becomes: Can you find a generally covariant function that characterizes the tendency of the gravitational field to become more inhomogeneous with time, a "gravitational entropy"?

However, in tackling this problem one immediately encounters a major conceptual difficulty: general relativity is a dynamical, not a thermodynamical theory, and it does not deal with temperatures. As prima facie evidence, I have been keeping a rough count of how many times the word "temperature" has arisen in the past four days and I would say five, \pm 1 , and in all cases it has referred to the cosmic microwave background. Another way of stating the difficulty is that general relativity deals with far-from-equilibrium systems for which a temperature is not well-defined. One usually, for example, doesn't talk about the temperature of a pendulum. Of course, you could define an effective temperature $1 / 2 m v^{2} \sim k T$ but the fact remains-

Jurgen Ehlers: The pendulum is not in equilibrium. 


\section{TR: Exactly.}

So the question remains whether the concept of entropy can be incorporated into GR and how to do it. There don't seem to have been too many attempts in the literature to define a gravitational entropy. The most well-known is Roger Penrose's Weyl-tensor hypothesis [1], the idea being that the square of the Weyl-tensor should be zero at the Big Bang but increase monotonically thereafter. However, the work of Bonnor [2], as well as that of Wainright and collaborators [3, 因, 5], have thrown some doubt on the proposal. It's not my intent to criticize the hypothesis but rather to rethink the problem. Smolin [6] and $\mathrm{Hu}$ and Kandrup [7] have examined some aspects of gravitational entropy, but not the arrow-of-time question. After I began work on this topic a paper by Brandenburger et al. appeared [8], which again is concerned with the thermodynamical aspects of the entropy of gravitational radiation, but in the places where we can compare our work with their's, it seems to agree. Most of this talk will be based on a paper I wrote with Peter Anninos that appeared in Phys Rev D [9].

Roger Penrose: By way of clarification I should say that I never meant the Weyl tensor to be a measure of gravitational entropy. I merely wanted it to be zero at the big bang.

TR: Really? Well, if that's true I apologize.

Roger Penrose: You're not the only person to have that misunderstanding.

TR: It does seem to be widespread. Perhaps we can sort out where people got this idea.

At any rate, I decided to tackle the problem by a direct statisticalmechanics approach. We examine the definition

$$
S=\ln \Omega
$$


where $S=$ gravitational entropy and $\Omega=$ volume of phase space for the field.

Why choose such a definition? In thermodynamic systems the phase space is much too hard to calculate. Instead you generally evaluate the partition function $Z \equiv \sum_{i} e^{-\beta E_{i}}$, from which the entropy is readily derived(here $\beta \equiv 1 /(T)$ and $\bar{E}=$ the mean energy.) But this requires a temperature, which we don't have.

On the other hand, consider a collection of $N$ harmonic oscillators with the usual Hamiltonian

$$
H=\frac{1}{2} \sum_{i=1}^{N} \dot{\phi}_{i}^{2}+\frac{k}{2} \sum_{i=1}^{N} \phi_{i}^{2}
$$

This has a phase space. In fact, for one pendulum you know the phase space: an ellipse. If you increased the energy of the pendulum it would describe a slightly larger ellipse and the logarithm of the area between the two ellipses could be taken as entropy. However,we don't talk about entropy of dynamical systems-you know exactly the trajectory of the pendulum and so it doesn't make sense to define the logarithm of the area as entropy. You need an ergodic system, some lack of information.

However, if we consider a system of oscillators with random phases, then we don't know where the oscillators are in this $2 \mathrm{~N}$-dimensional phase space and it apparently makes sense to interpret the logarithm of this volume as entropy. In essence I am going to model the field as a collection of oscillators and treat the oscillators as a microcanonical ensemble, in which you assume any region of phase space is occupied with equal probability. In that case $S=\ln \Omega$ is equivalent to $S=-\sum_{i} p_{i} \ln p_{i}$.

To give you an idea of how it works, for the Hamltonian given in (2) above, we can actually calculate the phase-space volume analytically, using beta-functions, much in the way you'd calculate the volume of a sphere in elementary calculus. The result 
is:

$$
\Omega=\frac{(2 \pi)^{N} H^{N}}{k^{N / 2} N !}
$$

Noting that $H=R^{2}$, this indeed is the volume of a $2 \mathrm{~N}$-dimensional sphere, or more precisely an ellipsoid. Taking $S=\ln \Omega \approx N$ one can recover from this formula in the appropriate limits :

- Entropy of an ideal gas $(V=k=0)$

- Classical limit of entropy of an Einstein solid $(H=E=N T)$

This isn't too surprising, as Einstein modeled a solid as a collection of harmonic oscillators. You can also get approximately

- Entropy of the electromagnetic field.

In fact you can write down

- Partition function $\left(\frac{2 \pi}{\beta \omega}\right)$

although I haven't figured out what to do with it.

You might well be asking what this has to do with the gravitational field. The first reason to examine the above definition of entropy is that it does not require a temperature. The second reason is that there exists a Hamiltonian formulation of gravity (the ADM formulation). One can write down Hamiltonians for gravitational systems and thus one should be able to define $\Omega$ below $H$.

Here one needs to make an important caveat: In general, $H$ will be time dependent, and therefore not the energy. So this is a slight extension of the usual statistical mechanics definition of energy. This doesn't necessarily bother me. In stat mech you for a system in contact with a heat reservoir the change in entropy is $\Delta S=\Delta Q / T$. If you believe that $Q$ is the kinetic energy of the Hamiltonian of the system, this does assume a change in $H$, although a quasi-static one. A time-dependent $H$ 
is characteristic of an open system, which is really what we have in GR, and it is this time-dependence that will cause a change in entropy. So how do we calculate the change in $H$ ?

Well, finally, I'll write down a metric, one for gravitational-wave perturbations:

$$
d s^{2}=a^{2}(\eta)\left[-d \eta^{2}+\left(\delta_{i j}+h_{i j}(\eta, z)\right) d x^{i} d x^{j}\right],
$$

Here $\eta=$ conformal time; $a(\eta)=$ expansion scale factor;

and $h_{i j} \ll \delta_{i j}=$ represent the gravitational wave perturbations.

Assuming a single polarization, the Einstein-Hilbert action to second order in the perturbation variables is:

$$
I=\frac{1}{64 \pi} \int a^{2}\left(\dot{h}^{2}-h^{\prime 2}\right) d^{4} x,
$$

Here $(\cdot) \equiv d / d \eta$ and $\left(^{\prime}\right) \equiv d / d z$.

It's sometimes useful, in particular when comparing with other work, to change variables to $\phi \equiv a h / \sqrt{32 \pi}$, and you get for the above Lagrangian density:

$$
\mathcal{L}=\frac{1}{2}\left[\dot{\phi}^{2}-\phi^{\prime 2}+\frac{\ddot{a}}{a} \phi^{2}\right]
$$

The corresponding Hamiltonian density is by definition $\mathcal{H} \equiv$ $\pi \dot{q}-\mathcal{L}=$, or in this case,

$$
\mathcal{H}=\frac{1}{2}\left[\dot{\phi}^{2}+\phi^{\prime 2}-\frac{\ddot{a}}{a} \phi^{2}\right] .
$$

The Hamiltonian is $H=\int \mathcal{H} d^{3} x$. In our case we want to make contact with the system of $\mathrm{N}$ harmonic oscillators and so we discretize the integral:

$$
\bar{H}=\frac{1}{2} \sum_{i=1}^{N}\left(\bar{\pi}_{i}^{2}+\bar{\phi}_{i}^{2}-\frac{\ddot{a}}{a} \bar{\phi}_{i}^{2}\right),
$$


where $\bar{\pi}=\pi(L / N)^{3 / 2}, \bar{\phi}=\phi(L / N)^{3 / 2}, \bar{H}=H / N^{2}$, and L is an arbitrary length scale.

Notice that this equation resembles the harmonic oscillator Hamiltonian Eq (2) except for two features. The first is that it contains a gradient term: $\phi^{\prime}=\phi_{i}-\phi_{(i+1)}$. This is not a big problem. By defining a new variable $\xi_{i} \equiv \phi_{i}-\phi_{(i+1)}$, one can integrate this "nearest-neighbor potential" in the same way one does the harmonic oscillator potential and get an result for the phase space identical to Eq (3), but for insignificant numerical factors.

The second feature is the main problem: the minus sign in front of the harmonic-like potential of the last term. This implies, first, that $H$ can change sign and that, second, we have a reflection barrier instead of a potential well. This implies that the phase space is unbounded. Without going into the gory details, I claim that in the perturbative limit, one can still analytically integrate the phase space using hypergeometric functions (see Rothman and Anninos for specifics), and one formally recovers expression (3). Nevertheless, one is forced to impose some sort of cutoff, in momentum space if $H>0$ or in configurations space if $H<0$. (It turns out that $H>0$ represents growing modes and $H<0$ represents decaying modes, which is an interesting result in its own right, but we do not go into details here. The growing modes, which result in an increase in entropy, are of the main concern.) There are several conceivable methods to impose the necessary cutoffs, but it turns out they all give qualitatively the same results.

So, the procedure to calculate the entropy is to regard $\Omega$ as constant on each spacelike hypersurface, in which case we can take $\ddot{a} / a=k$, the spring constant. To find $\Omega$, we first need $H$ , which we get by considering the equations of motion resulting 
from the action (5):

$$
\ddot{h}+2 \frac{\dot{a}}{a} \dot{h}-h^{\prime \prime}=0
$$

We assume a separable solution with random phases $\alpha_{j}$ :

$$
\sum_{j}\left(\ddot{h}_{j}+\frac{2 \dot{a}}{a} \dot{h}_{j}-h_{j}^{\prime \prime}\right)=\left(\ddot{h}+\frac{2 \dot{a}}{a} \dot{h}-h^{\prime \prime}\right) \sum_{j} e^{i \alpha_{j}}=0,
$$

The subscript $j$ refers to different waves, not to different coordinates. By taking the phases to be random we are essentially assuming an incoherent source, and since the $\alpha_{j}$ do not enter into the solutions, I supress them from now on. Eq (9) is Bessel's equation and for the matter-dominated epoch with $a=a_{o} \eta^{2}$, one gets:

$$
h \propto \eta^{-3 / 2} J_{ \pm 3 / 2}(k \eta) e^{i k z}
$$

Now, the $J$ 's have standard asymptotic forms for $k \eta \ll 1(\lambda \gg$ Hubble radius )and $k \eta \gg 1$ ( $\lambda \ll$ Hubble radius).

For $k \eta \ll 1$, we have:

$$
h=\left[h_{1}(k \eta)^{-3}+h_{2}\right] e^{i k z},
$$

where $h_{1}$ and $h_{2}$ are constants representing the decaying and growing modes respectively. (In this case the "growing" modes are constant in time.)

For $k \eta \gg 1$ we have:

$$
\begin{gathered}
h \propto \sqrt{\frac{2 k^{3}}{\pi}}\left(\frac{1}{k \eta}\right)^{2}[\cos (k \eta)+\sin (k \eta)] e^{i k z} \\
\propto(k \eta)^{-2} \times[\text { oscillations }]
\end{gathered}
$$

In terms of $\phi \sim a h$, for $k \eta \gg 1$ :

$$
H \propto \pi^{2}+k^{2} \phi^{2}
$$

$=$ Harmonic oscillator potential at fixed time, 
and

$$
\Omega \propto \frac{H^{N}}{k^{N / 2}} \propto \text { constant } \times[\text { oscillations }]
$$

Therefore $\Omega$ oscillates, but otherwise does not change. Given that in this limit $H$ is a harmonic oscillator, this isn't too surprising. One could average over several cycles to obtain a strictly constant $H$ :

$$
{ }^{(4)} \mathcal{H}=\int d^{4} x\left[\pi^{2}+k^{2} \phi^{2}-\frac{\ddot{a}}{a} \phi^{2}\right] \text {. }
$$

In the nonlinear regime, $\Omega$ would increase, which is encouraging for the interpretation of $\ln \Omega$ as entropy.

In the opposite limit $k \eta \ll 1$, the gradient term in (8) is negligible and we get for the time dependence of $H: H \propto \pi^{2}-\ddot{a} \phi^{2} / a$ and $\Omega \propto H^{N} /(\ddot{a} / a)^{N / 2}$.

$$
H \propto\left\{\begin{array} { l } 
{ \eta ^ { 2 } , } \\
{ \eta ^ { - 4 } , }
\end{array} , \text { and } \Omega \propto \left\{\begin{array}{ll}
\eta^{3 N} & , \text { for growing modes } \\
\eta^{-3 N} & , \text { for decaying modes }
\end{array}\right.\right.
$$

This result is at first glance somewhat perplexing. Superhorizon modes should be frozen in, no oscillations allowed, so why should we get an increase in $\Omega$ ? In terms of $h$ one sees that indeed: $\dot{\phi}=\dot{a} h+a \dot{h}=\dot{a} h$. That is since $\dot{h}=0$, the superhorizon modes are frozen in and the increase in $\Omega$ is due entirely to expansion $\dot{a}$. Furthermore, since the superhorizon modes are frozen, we know the position of each oscillator and the assumption of random phases breaks down. This suggests that our definition of entropy is only applicable to subhorizon processes, which is not necessarily a bad thing. Such an interpretation agrees with the conclusions of Brandenburger el al.

Without going into further details, I merely state that one can repeat the above analysis for density perturbations (radiation 
and dust) in flat, open and closed cosmologies. One always has to worry about gauge problems in inhomogeneous models and so we use the gauge-invariant formalism of Mukhanov et al. [10]. We find that in all cases $\Omega$ monotonically increases for growing modes $(H>0)$ and decreases for decaying modes $(H<0)$. This is of course encouraging for the interpretation of $\ln \Omega$ as a measure of gravitational entropy.

In one of George's preprints [11] he and his coauthor Reza Tevakol mentioned that it is hard to see how to apply this formalism to GR in full generality since the number of modes, $N$, is infinite in field theory. I should clear up this misunderstanding. We are not doing a second-quantized field theory. $N$ is merely the number of Planckian oscillators- modes in a box-which should be finite if the box under consideration is of finite size. To be sure, $N$ is merely the Jeans' number $\sim \omega^{3} a^{3}$, where $\omega$ is the oscillator frequency. In all our models $S \sim N$ (see especially Eq (26) below), as is the case for electromagnetic radiation.

From this fact, we can actually calculate the entropy density of gravitational radiation produced during inflation. I did this calculation quite recently and it is not to be found in Rothman and Anninos, but it does follow closely the method outlined by Peebles [12] on page 492-494. Our Lagrangian density can be rewritten slightly as

$$
\mathcal{L}=m_{p}^{2}\left(\dot{h}^{2}-h^{\prime 2} / a^{2}\right)
$$

where now ${ }^{\circ}=d / d t$. This is a fairly generic Lagrangian density with $m_{p} \dot{h}$ playing the role of the usual kinetic energy $\dot{\phi}^{2}$ term. As Peebles shows, $m_{p} h \sim H$, the Hubble constant, is the rms value of the field. If we assume that $h$ represents the size of the perturbation during inflation, we then have

$$
h \sim \frac{H}{m_{p}}=\frac{8 \pi}{3} \frac{\epsilon^{2}}{m_{p}^{2}},
$$


where $\epsilon$ is the energy scale of inflation.

Now, as mentioned above, the entropy $S \sim N$. Define an entropy density $\sigma \equiv N / a^{3}=\omega^{3}$. But in our case, $\omega \sim H \sim m_{p} h$ and so $\sigma \sim h^{3} m_{p}^{3}$. All we need to do is scale this down to the present day to find $\sigma_{o} \sim h_{o}^{3} m_{p}^{3}$, where $h_{o}$ is today's value of $h$. Since $\omega \sim m_{p} h$, we can also write $\sigma_{o} \sim\left(m_{p} h_{o} \omega_{o}\right)^{3 / 2}$. Now, $h_{o}$ is the strain that has just started oscillating as its corresponding proper wavelength $\lambda_{o}$ falls within the Hubble radius. Peebles shows that

$$
h_{o} \sim \Omega_{r}^{1 / 2} \lambda_{o} H_{o} \frac{\epsilon^{2}}{m_{p}^{2}}
$$

But $\lambda_{o} \sim 1 / \omega_{o}$. Thus

$$
\sigma_{o} \sim \Omega_{r}^{3 / 4} H_{o}^{3 / 2} \frac{\epsilon^{3}}{m_{p}^{3 / 2}}
$$

With $H_{o} \sim T_{o}^{4} / m_{p}^{2}$, we get

$$
\sigma_{o} \sim \Omega_{r}^{3 / 4} T_{o}^{3} \frac{\epsilon^{3}}{m_{p}^{3}} .
$$

This says that the entropy of gravitational radiation produced during inflation is supressed from that of the microwave background by the factor $\epsilon^{3} / m_{p}^{3}$. Apart from the factor $\Omega_{r}^{3 / 4}$, which is of order unity, Eq (22) is identical to an equation in Brandenburger et al., obtained by much more difficult means.

To conclude this part of the talk, we find that the candidate gravitational entropy function $S=\ln \Omega$ behaves reasonably on subhorizon scales in that it increases monotonically, and when applied to the case of gravitational radiation during inflation produces the result that a generic field theory should produce. Nonlinear calculations should be carried out. 
I would like now to briefly discuss two other aspects of this project. We did compare the behavior of $S$ with that of $C^{2}$ for the metric

$$
d s^{2}=a^{2}(\eta)\left[-(1+2 \Phi(\eta, z)) d \eta^{2}+(1-2 \Phi(\eta, z)) \gamma_{i j} d x^{i} d x^{j}\right]
$$

where $\Phi$ is the gauge-invariant version of $h$ with solution $\Phi=$ $\left(\bar{u}_{1}+\bar{u}_{2} \eta^{-5}\right) e^{i(k z)}$. Specifically, we examined

$$
C_{\alpha \beta}^{\gamma \delta} C_{\gamma \delta}^{\alpha \beta}=\frac{16\left(\Phi_{, z z}\right)^{2}}{3 a^{4}}
$$

where the solution for $\Phi$ is the one just given. I bear in mind Roger's earlier comment that he did not mean for $C^{2}$ to be a measure of entropy, but with $a \sim \eta^{2}$, we find that (24) decreases with time and inhomogeneity, which seems a rather strange behavior. You might think to correct this behavior by introducing an overall minus sign, but then the decaying modes increase with decreasing inhomogeneity, which is no less strange.

On the other hand, we also examined the form of the Penrose hypothesis suggested by Wainwright and collaborators [3, 4, 5] To lowest order this is:

$$
\frac{C_{\alpha \beta}{ }^{\gamma \delta} C_{\gamma \delta}^{\alpha \beta}}{R_{\alpha \beta} R^{\alpha \beta}}=\frac{4 a^{4}\left(\Phi_{, z z}\right)^{2}}{9\left(\dot{a}^{4}-a \dot{a}^{2} \ddot{a}+a^{2} \ddot{a}^{2}\right)}=\frac{\eta^{4}\left(\Phi_{, z z}\right)^{2}}{27},
$$

which has a time dependence

$$
\frac{C_{\alpha \beta}^{\gamma \delta} C_{\gamma \delta}^{\alpha \beta}}{R_{\alpha \beta} R^{\alpha \beta}} \propto \begin{cases}\eta^{4} & , \quad \text { for growing modes } \\ \eta^{-6}, & \text { for decaying modes. }\end{cases}
$$

Such a time dependence is not only reasonable but is, to one's great surprise (at least our great surprise), identical to that of the Hamiltonian $H$ for $k=0$ dust. The expression (25) is an approximation, so we don't know whether we are facing a coincidence or something more profound. We haven't investigated 
the matter further, but I think if there is a graduate student interested in numerical work, it might be worth looking into.

I'd like now to turn to my recent-and so far not entirely successfulattempt to apply this formalism to black holes. Take expression (3) for the phase space of $N$ harmonic oscillators. Now imagine creating a black hole out of quantum oscillators. In this case the Hamiltonian $H$ should be the total energy, $M$ and we should also have $M=N \bar{\epsilon}$, where $\bar{\epsilon}=$ average oscillator energy. Further the angular frequency $\omega=2 \pi \bar{\epsilon}=k^{2}$, where $k$ is the spring constant. Plugging all this into (3) and using Stirling's approximation on the $N$ ! gives identically

$$
\Omega=e^{N} \text { and } S=N
$$

Again , $N=M / \bar{\epsilon}=M \lambda$, where $\lambda$ is the wavelength. A priori, we expect $\lambda \approx 4 M$. With this value we find

$$
S \approx 4 M^{2}=S_{B H} /\left(2 \pi^{2}\right)
$$

where $S_{B H}$ is the canonical Bekenstein-Hawking value.

About half the people I have shown this to call it a "discovery" and the other half call it a "dimensional coincidence." I think it is bit more than the latter. In his review article on string theory and black holes, Gary Horowitz 14 points out that string entropy is given by $S_{s} \sim \ell_{s} M_{s}$, where $\ell_{s}$ is the length of the string and $M_{s}$ is the string mass. In the limit that $\ell_{s}$ becomes the Schwarzschild radius and $M_{s}$ becomes the mass of the black hole, we get $S \sim R_{B H} M_{B H}$. Horowitz calls this "remarkable," yet it seems to me to be essentially the same argument I just gave for ordinary oscillators. The result appears to be quite general.

The main problem with this technique, as I see it, is that it doesn't give a value for $N$, which must be put in by hand. The 
question is, if you believe $S \sim N$, can you get a better fix on $N$ ? The assumption that black-hole entropy is related to the number of interior modes has been challenged, but in light of the recent developments in string theory, it seems to me worth the effort to find a more modest description along these lines. What I've recently been attempting to do is count interior modes of a Schwarzschild black hole.

The idea is fairly simple. Consider the usual scalar wave equation on a Schwarzschild background:

$$
\begin{aligned}
& -\left(1-\frac{2 M}{r}\right)\left[\left(1-\frac{2 M}{r}\right) \psi_{, r r}+\frac{2 M}{r^{2}} \psi_{, r}\right] \\
& +\left\{\left(1-\frac{2 M}{r}\right)\left[\frac{2 M}{r^{3}}+\frac{\ell(\ell+1)}{r^{2}}\right]-\omega^{2}\right\} \psi=0
\end{aligned}
$$

This has a well-known effective potential (see MTW, p. 868 [13), which goes to minus infinity at the origin. But the only thing that happens at $R=2 M$ is that $V_{\text {eff }}$ goes to zero. Moreover, although the Legendre-functions $\left(P^{\prime} s\right.$ and $\left.Q^{\prime} s\right)$ that are the solutions to (28) in the limit of zero frequency blow up either at $2 M$ or infinity (the no-hair theorem), the $P^{\prime} s$ are actually regular on the interior. So the idea is to put in a Planck-frequency cutoff $\equiv \omega_{p}$ and see if one can count modes in a sensible fashion.

Now, you might think this is impossible, since (28) has no analytic solutions on the interior, but you don't actually need to solve the equation. Define a new variable related to the old by the integrating factor of the equation: $\psi=\left(\frac{2 M-r}{r}\right)^{-1 / 2} \bar{\psi}$. This eliminates the first derivative term in the wave equation. Recalling that $r$ is timelike for $r<2 M$, make the ansatz $\bar{\psi} \sim e^{i \omega r}$. Then you find

$$
\frac{d^{2} \bar{\psi}}{d t^{2}}+\left[\omega^{2}\left(\frac{2 M-r}{r}\right)^{2}-\frac{M^{2}}{r^{4}}-\left(\frac{2 M-r}{r}\right) \frac{\ell(\ell+1)}{r^{2}}\right] \bar{\psi}=0
$$

Since $t$ is spacelike for $r<2 M$, this is just like a flat-space wave 
equation,

$$
\bar{\psi}^{\prime \prime}+k_{\text {eff }}^{2} \bar{\psi}=0,
$$

where $k_{\text {eff }}^{2}$ is the quantity in brackets in (29).

Now you just count modes in a box. Following the suggestion of 't Hooft [15], the total number of radial modes beneath a frequency $\omega$ will be,

$$
N_{\omega}=\frac{1}{\pi} \int_{r, \ell} k_{e f f} d \ell d r
$$

I claim that you can integrate this expression. The integrals converge at $R=2 M$ and the potential terms don't contribute. The only thing that contributes is the cutoff frequency and the radial cutoff $(=2 M)$. In fact, it is quite remarkable that of the six terms in the final integral, the only non-negligible term is the one with the correct scaling; and the result is $S=(3 / 8) \pi \omega^{2} M^{2}$. Choosing the Planck frequency $\omega_{p}=2 \pi$ as the cutoff, the expression becomes $S=(3 / 16) \pi S_{B H}$, or about .6 the canonical value. In flat space, one gets exactly $.25 S_{B H}$.

Now, a scalar wave has only one polarization. If one counted gravitational perturbations instead, one would use the Zerilli or Regge-Wheeler equation, which have different potentials. But since the potential terms don't contribute, one gets the same answer per mode. Therefore, for the two modes of gravitational waves, the result is $1.2 S_{B H}$, which seems too close for comfort.

Unfortunately, there are two flies in the ointment. The first is that the integral over $r$ in (31) is a timelike integral, which makes the interpretation unclear. More seriously, I have ignored azimuthal modes. To count them, the integrand in (31) should contain a factor $(2 \ell+1)$. However, in that case the scaling goes wrong; i.e., one gets $S \sim w^{3} r^{3}$.

I am at this time unable to justify ignoring the azimuthal modes and so I must let the result stand as what may well be a dimen- 
sional fluke. Even so, it contains some interesting points in common with earlier work of Bombelli et al. [16]. They calculate the entropy due to a system of coupled oscillators. To carry out the calculation they define a density matrix and perform partial traces over an imaginary sphere that separates "inside oscillators" from "outside oscillators." In the continuum limit they find that the entropy of the oscillators within this imaginary sphere scales as the area. Although the considerations by which they obtain their results are somewhat different than mine, they nevertheless find that the result is independent of the potential and depends only on the cutoffs in $\omega$ and $r$ and is proportional to the number of fields under consideration. (This will be true whether azimuthal modes are considered or not.) Whether this points to some deeper understanding of black hole entropy or is merely a reflection of the fact that both calculations are based on oscillator systems, or whether it is a basic reflection of the dimensionful constants that govern the problem is something that I have yet to sort out. Thank you.

\section{References}

[1] Penrose, R. (1989), in Fourteenth Texas Symposium on Relativistic Astrophysics, ed. E. J. Fenyves (New York Academy of Sciences, New York).

[2] Bonnor, W.B. (1987) Physics Letts. A 122, 305.

[3] Wainwright, J. and Anderson, P.J. (1984), Gen. Rel. Grav. 16,609 .

[4] Goode, S.W. and Wainwright, J. (1985), Class. Quantum Grav. 2, 99. 
[5] Goode, S.W., Coley, A.A. and Wainwright, J. (1992), Class. Quantum Grav. 9, 445.

[6] Smolin, L. (1985) Gen. Rel. Grav. 17, 417.

[7] Hu, B. and Kandrup, H. (1987) Phys. Rev. D 35, 1776.

[8] Brandenberger, R., Mukhanov, V. and Prokopec, T. (1993) Phys. Rev. D 48, 2443.

[9] Rothman, T. and Anninos, A. (1997) Phys. Rev. D 55, 1948.

[10] Mukhanov, V., Feldman, H. and Brandenberger, R. (1992) Phys. Rep. 215, 203.

[11] Tavakol, R. and Ellis, G.F.R., "On Gravitational Entropy," University of Cape Town, preprint, (1997).

[12] Peebles, P.J.E., Principles of Physical Cosmology(Princeton: Princeton University Press, 1993.

[13] Misner, C.W., Thorne, K.S. and Wheeler, J.A. (1973), Gravitation, (W.H. Freeman and Company, New York).

[14] Horowitz, Gary, gr-qc/9704072

[15] 't Hooft, G. (1986), Nuc. Phys. B 256, 727.

[16] Bombelli, L. et al. (1986), Phys. Rev D 34, 373. 